\title{
Asociatividad y transferencia de tecnología: caso de articulación público-privada en Argentina
}

\author{
Darío Gabriel Codner*
}

Recibido: 1 de junio de 2011 Evaluado: 14 de julio de 2011 Aceptado: 29 de julio de 2011

\section{Resumen}

En las nuevas formas de producción del conocimiento, la innovación se constituye en mecanismo para la competitividad. También diferentes corrientes económicas destacan el papel de la innovación en el desarrollo. Los sistemas de innovación, las infraestructuras, la formación de recursos humanos, las normas regulatorias, entre otros, son aspectos determinantes. Además, hoy en día se rescatan las redes, la asociatividad y los consorcios como nuevas formas de producción de conocimiento cooperativo entre una alta diversidad de actores. Este artículo trata estas nuevas realidades, a partir de un caso de asociación público-privada en el campo de la biotecnología.

\section{Palabras clave}

Innovación, asociatividad público-privada, transferencia tecnológica, consorcio, producción de conocimiento. 


\title{
Partnerships and technology transfer: case of public-private articulation in Argentina
}

\author{
Darío Gabriel Codner
}

\begin{abstract}
In the new forms of knowledge production, innovation constitutes a mechanism for competition. Also, different economic trends highlight the role of innovation in development. The innovation systems, infrastructures, training of human resources, regulatory standards, among others, are key aspects. In addition, today networks, partnerships and trusts are relevant as new forms of knowledge production in a cooperative manner between a high diversity of stakeholders. This article addresses these new realities, from a case of public-private partnership in the field of biotechnology.
\end{abstract} Aceptado: 29 de julio de 2011

\section{KeYwORDS}

Innovation, public-private partnership, technology transfer, consortium, knowledge production. 


\section{INTRODUCCIÓN}

Los últimos años se han caracterizado por el acelerado proceso transformador de la sociedad en sus dimensiones culturales, económicas, políticas y sociales. La noción "sociedad del conocimiento" resalta nuevas formas de producción, en las cuales el conocimiento justamente se constituye en uno de los principales factores explicativos del crecimiento económico, junto con el capital y el trabajo.

En cuanto a lo económico, la innovación se convierte, en términos generales, en la actividad dominante, y sus fuentes se hacen más difusas mientras las organizaciones se reconfiguran en su búsqueda como mecanismo para desarrollar competitividad (David y Foray, 2002). Según el Manual de Oslo de la OCDE (2005), por innovación se entiende la implementación en el lugar de trabajo o en las relaciones externas de un producto (bien o servicio), de un proceso nuevo o con un alto grado de mejora, de un nuevo método de comercialización u organización aplicado en las prácticas de negocio.

Específicamente, desde las diferentes corrientes económicas se coincide en el papel de la innovación para el desarrollo (Nelson y Winter, 1982; Nelson,1993; Lundvall, 1985; Teubal, 1996: 7; Freeman, 1982, 1987, entre otros). El punto de partida común del conjunto de estas investigaciones es el abandono de la concepción de la innovación como un proceso de decisión individual independiente del contexto, en beneficio de una concepción de actores insertos en distintas redes de instituciones.

La asociación entre capacidades tecnológicas y procesos de innovación y los agentes que se desempeñan según incentivos del mercado, del Estado, de las empresas y de las instituciones impulsa la conceptualización del Sistema Nacional de Innovación (SNI) como paradigma en el diseño de políticas científicas, tecnológicas y de innovación. Este concepto introducido por Freeman (1982, 1987) y Lundvall (1985) enfatiza sobre el carácter interactivo de la producción y de la innovación, al igual que sobre la importancia y complementariedad entre las innovaciones radicales, técnicas y organizacionales.

Diferentes esfuerzos conceptuales se han realizado para describir los procesos de innovación, principalmente en el marco de una dinámica de interacción compleja entre gobierno, academia e industria (Sábato, 1970; Etzkowitz y Botana, 1997). Así, las políticas de innovación se basan en el desarrollo de un "clima" que estimule el desarrollo de innovaciones, a partir de la provisión de infraestructura científica y tecnológica, estrategias de formación de recursos humanos, apoyo directo o indirecto a empresas, desarrollos normativos y regulatorios, entre otros aspectos.

Por otro lado, se concede una relevancia fundamental a la creación de productos y servicios intensivos de conocimiento. Este escenario es resultado de una nueva forma de producir conocimiento, donde la expectativa de aplicación de los resultados de investigación y desarrollo se estabiliza en la agenda de las academias (Gibbons, 1997). De este modo, se determina un nuevo patrón basado en la búsqueda de resultados prácticos, en problemas complejos resueltos interdisciplinariamente y en la adopción de nuevas formas organizativas con alta diversidad de actores. 
En los últimos años se ha comenzado a acuñar una nueva categoría conceptual que describe la cooperación entre los grupos de investigación y las compañías: la innovación abierta (Chesbrough, 2003). Particularmente en el sector biotecnológico ${ }^{1}$ este concepto adquirió gran difusión, puesto que permitió diluir límites de la llamada ciencia básica, aplicada y tecnológica. Este fenómeno se manifiesta comparando diferentes sectores tecnológicos. Por ejemplo, las más importantes compañías biotecnológicas producen conocimiento científico y tienen origen en los sistemas universitarios de I+D (Pisano, 2006). Además, se trata de un sector multiparadigmático en lo tecnológico, es decir, un sector en el que se desarrollan diferentes tecnologías que no compiten entre sí, permitiendo que no exista liderazgo o monopolios tecnológicos. Así, las empresas conviven en nichos de mercado dominando diferentes tecnologías.

Lo anterior obliga a las empresas a buscar asociaciones con grupos de I+D para poder dominar los mercados, puesto que nuevas tecnologías podrían originarse en laboratorios de investigación y permitir el desarrollo de nuevos competidores o productos sustitutos, tal como se puede apreciar analizando el sector productor de medicamentos que está "mutando" hacia desarrollos de base biotecnológica moderna, sobre el fundamento de conocimiento científico desarrollado en universidades e institutos de investigación.

La generación de redes asociativas entre el ámbito público y privado emerge como

1 La biotecnología moderna es la aplicación de los resultados de las investigaciones en biología molecular, genética molecular y ciencias biológicas en general, para la fabricación de medicamentos, vacunas, alimentos, nuevos materiales, etc. una realidad en las universidades latinoamericanas. Así, el presente trabajo describe un caso de asociación público-privada en el campo de la biotecnología, al igual que su desenvolvimiento y efectos.

\section{LAS CAPACIDADES DE INVESTIGACIÓN CIENTÍFICA COMO FACTOR ESENCIAL PARA LA TRANSFERENCIA TECNOLÓGICA}

La Universidad Nacional de Quilmes (UNQ) desde sus orígenes en 1989 se ha destacado por el desarrollo de su perfil académico con base en el reclutamiento de jóvenes investigadores. De este modo, la universidad institucionaliza su Departamento de Ciencia y Tecnología con un fuerte sesgo hacia la investigación biotecnológica. En ese marco, desde 1996 se inaugura y desarrolla el Laboratorio de Oncología Molecular, desde el cual se han originado solicitudes de varias patentes, algunas de las cuales han sido concedidas, licenciadas y comercializadas. Esto es resultado de una sostenida producción científica de alta calidad medida a través del factor de impacto de sus publicaciones, las que hacen referencia a sus trabajos en patentes extranjeras.

Hace diez años una empresa farmacéutica (en adelante la empresa) se enfrentó a necesidades de investigación y desarrollo. Específicamente quiso estudiar in vitro $\mathrm{y}$ en modelos animales preclínicos productos que estaban en fase de investigación y desarrollo. Inició entonces una fuerte estrategia de establecimiento de acuerdos de investigación científica con laboratorios universitarios.

Con una interesante estrategia, la empresa se vinculó a centros cubanos de 
investigación en biotecnología, con el objeto de probar algunos desarrollos en vacunas oncológicas. En ese contexto, para obtener algunos servicios tecnológicos, la empresa recurrió también al Laboratorio de Oncología Molecular de la Universidad Nacional de Quilmes. En breve, los investigadores principales del laboratorio universitario se constituyeron en interlocutores y enlace entre el grupo de investigación cubano y las empresas. Así se fue construyendo un marco de confianza entre los directivos empresarios y los investigadores del Laboratorio de Oncología Molecular, lo que derivó en efectos inesperados.

Esta relación de confianza fue clave para que los investigadores le ofrecieran a la empresa la licencia de una patente sobre un desarrollo de propiedad de la Universidad Nacional de Quilmes en tratamientos en oncología, en otros desarrollos biotecnológicos para estudio y en el tratamiento del cáncer. Tales invenciones tenían posibilidad de ser patentadas y fueron probadas en la universidad en animales de laboratorio, con el objetivo claro de atender la enfermedad en humanos.

La empresa propuso realizar estudios preclínicos y farmacológicos en animales de mayor tamaño, puesto que existía la potencial aplicación en medicina veterinaria. Se apuesta, entonces, al desarrollo de ese producto en el mercado veterinario nacional e internacional.

Así se concretó una formulación peptídica hemostática y antitumoral para ser aplicada durante la extirpación quirúrgica de tumores sólidos en mascotas. Este nuevo producto se patenta internacionalmente $\mathrm{y}$ se licencia a una compañía para su explo- tación comercial en todo el mundo en el ámbito veterinario. Actualmente, el desarrollo se encuentra a la venta en Argentina con el nombre comercial de "Desmopresina Biogénesis-Bagó" y existen compañías interesadas en el extranjero². Durante el 2010 la Universidad Nacional de Quilmes recibió, por primera vez desde su creación, regalías por la venta de dicho producto.

En cuanto a la invención para uso humano, el mismo se encuentra actualmente en estudios clínicos de fase III en el mundo, con alta probabilidad de obtener la aprobación de las instituciones regulatorias. Dichos estudios clínicos están siendo llevados a cabo por la empresa.

De esta manera, en el marco de un diálogo abierto se fueron estableciendo las estrategias innovadoras y la detección de oportunidades desde la empresa, influyendo de este modo en la agenda de investigación del Laboratorio de Oncología Molecular ${ }^{3}$. Este proceso de aprendizaje mutuo fue incidiendo en el lineamiento de las investigaciones, sin que se afectara la libertad de los científicos, y facilitó, incluso, la cooperación internacional académica de los investigadores, al igual que la generación de papers, que fueron publicados con independencia de la empresa y mantuvieron criterios libres de temas de investigación.

2 El producto está siendo comercializado en Argentina por Biogénesis Bagó. Se trata de una droga indicada para ser utilizada como antitumoral y antimetastásico en cirugías de alto riesgo. Además, el producto ya está registrado en el extranjero, comenzando por países de Latinoamérica (como Nicaragua y Costa Rica que ya aprobaron el registro comercial).

3 Es interesante rescatar que los investigadores, en una primera instancia, no apreciaron la posibilidad de seguir con un desarrollo para uso veterinario, puesto que trabajaban en salud humana desde sus orígenes. De hecho, realizaron el ensayo en caninos, pensando que éste era otra "prueba de concepto" antes de encarar los ensayos clínicos en humanos. 


\section{NuEvos instrumentos DE FOMENTO DE LA ASOCIATIVIDAD PARA LA INNOVACIÓN: LA OPORTUNIDAD}

Las capacidades de investigación científica de calidad del laboratorio universitario, el perfil innovador de la empresa, el entendimiento y éxito en la transferencia tecnológica constituyeron el marco para el desarrollo de un consorcio.

En 2006 la Agencia Nacional de Promoción Científica y Tecnológica (ANPCyT), dependiente del Ministerio de Ciencia, Tecnología e Innovación Productiva, introdujo una iniciativa de financiamiento para asociaciones público-privadas cuyo objetivo fuera el desarrollo de conocimientos con potencial transferencia a la sociedad. De este modo, se crearon subsidios para Proyectos de Áreas Estratégicas (PAE).

Tales subsidios fueron introducidos como una nueva modalidad asociativa de apoyo a la investigación y tuvieron por objeto desarrollar "clusters de conocimientos" en áreas y temas prioritarios establecidos en el Plan Nacional de Ciencia Tecnología e Innovación.

De esta manera, la ANPCyT apoyó redes de grupos de investigación mediante múltiples proyectos que incluían desde la formación de recursos humanos hasta infraestructura y equipos científicos de tamaño medio. Los subsidios PAE se constituyeron, por tanto, en instrumentos dirigidos a la formación de consorcios de equipos de investigación, instituciones públicas y empresas, en pos de objetivos científicos y tecnológicos comunes.

Estos subsidios se implementaron a través de subproyectos integrados para lograr desarrollo del conocimiento, resultados en temas prioritarios, resolución de problemas esenciales y aprovechamiento de oportunidades emergentes en los sectores de producción de bienes y prestación de servicios. A partir de ello, los subsidios PAE introdujeron una complejidad intrínseca, dada la multiplicidad de actores y subproyectos; por ende, fueron el marco para el surgimiento de problemas de gobernanza sobre los proyectos, en cuanto a la administración de fondos, gerenciamiento, toma de decisiones y resolución de conflictos.

La publicidad del llamado a aplicar en subsidios PAE sucede a inicios del 2007, en el mismo tiempo en que la colaboración entre la empresa y el laboratorio evidenciaba ser un proceso sinérgico que simultáneamente aumentaba en complejidad.

Si bien la relación entre instituciones públicas -como universidades, institutos u hospitalesy empresas no tiene tradición en Argentina respecto a la transferencia de conocimientos y de tecnologías, no obstante la posibilidad de formular un proyecto de I+D asociativo se instaló en las agendas de la empresa y del laboratorio de investigación. El Laboratorio de Oncología Molecular y la empresa formularon conjuntamente un proyecto para aplicar al subsidio PAE en el campo de la investigación y del desarrollo de terapias y sistemas de diagnóstico molecular del cáncer.

Se formuló, entonces, el proyecto "Investigación y desarrollo de nuevas terapias y sistemas de diagnóstico de base molecular en cáncer". Los objetivos específicos de este proyecto fueron: a) la puesta a punto de modelos preclínicos de tumor para el estudio de mecanismos biológicos y de nuevos compuestos o protocolos antitumorales; b) la identificación y caracterización de nue- 
vos blancos moleculares de señalización en células tumorales agresivas; c) el desarrollo de nuevos compuestos peptídicos que estimulen la muerte celular o inhiban la vascularización de los tumores; d) el desarrollo de nuevos protocolos de inmunoterapia, a través de vacunas que vigoricen la respuesta específica antitumoral; e) el desarrollo de nuevos sistemas de detección molecular de alta sensibilidad para el seguimiento de la enfermedad residual.

A este proyecto se integraron, además, un hospital público, un instituto universitario de investigaciones, la Academia Nacional de Medicina y un grupo de investigación científica de la Universidad de Buenos Aires. La integración de los nuevos actores se fundamentó en la existencia previa de redes científicas de colaboración. Dicha red databa desde 1995 y se enmarcaba en una red internacional cuyo objetivo era el desarrollo de vacunas para patologías oncológicas.

\section{Asociatividad E IMPACTO}

La ejecución del proyecto asociativo público-privado generó nuevos desafíos y efectos de carácter sistémico e incidió de diferentes modos en las organizaciones. La formación del consorcio permitió la cohesión de grupos con distinta experiencia dentro del subsistema científico y tecnológico, facilitando la inclusión y aprendizaje de "actores inexpertos". El proyecto asociativo representó la posibilidad para que grupos de formación más reciente o sin experiencia dentro del sistema de ciencia y tecnología adquirieran capacidades de gestión de proyectos y pudieran incorporarse en el sistema científicotecnológico de promoción. Especialmente se facilitó el acceso a herramientas de fi- nanciación del sistema público y/o privado. Es decir, el proyecto asociativo traccionó a nuevos actores y, por ende, mejoró el carácter inclusivo de los instrumentos públicos de promoción de la innovación.

Por otro lado, el proyecto asociativo exigió la introducción de nuevas prácticas de gerenciamiento en $\mathrm{I}+\mathrm{D}$, debido a la variedad de instrumentos de promoción que componían los diferentes subproyectos y a la diversidad de intereses y marcos regulatorios de los actores. Así, siguiendo el modo 2 de Gibbons (1997), se demandó la búsqueda de acuerdos y nuevas formas de coordinación de proyectos en contextos de aplicación, buscando asegurar la eficacia en el logro de resultados y la transferencia de conocimientos y tecnologías.

En cuanto al efecto en el campo científico, la asociación entre diferentes integrantes del Sistema Nacional de Innovación ha sido significativo. Por un lado, los resultados han sido sinérgicos, es decir, se han logrado resultados de investigación y de transferencia tecnológica que de ningún otro modo hubiesen ocurrido sin la asociatividad del proyecto. El acceso a financiación y la formación del consorcio facilitó, sin duda, el abordaje de problemas más complejos y con mayores requerimientos. Además, el funcionamiento en red permitió la formación de profesionales en ámbitos interdisciplinarios que de otro modo no podrían formarse, puesto que se ha posibilitado la circulación y flujo de conocimientos y personas.

En cierto sentido, el subsidio se constituyó en un nuevo incentivo para la constitución de un consorcio de entidades públicas, hospitales y compañías privadas, cuyo impacto surge a priori como positivo en diferentes dimensiones. 
Este proceso, en el que se combinaron acciones del Estado con los intereses privados y científicos, favoreció notablemente la transferencia de conocimientos entre los grupos participantes. Por ejemplo, el hospital público que integró el consorcio mejoró en su capacidad de armar proyectos, así como en la relación de su capacidad de investigación y, muy especialmente, en su relación con la investigación clínica. También se estableció un servicio de diagnóstico molecular en un laboratorio del hospital, por una transferencia realizada desde la Universidad Nacional de Quilmes. A su vez, este nuevo laboratorio comenzó a ser referencia para otros grupos dentro del hospital, lo que potenció futuros trabajos de interés, como el desarrollo de un banco de tumores, por ejemplo.

La constitución del consorcio contribuyó, además, con la instalación de un laboratorio de proteómica de características únicas dentro de la universidad, lo que difícilmente podría haberse realizado sin el marco asociativo brindado por este proyecto.

Otro de los efectos generados por la constitución del consorcio fue el aumento de la escala. Por una parte, aumentó la visibilidad nacional e internacional y, por otro, se activó el acceso racional a equipamiento para la investigación, favoreciendo la interacción entre los proyectos y evitando la duplicación. En consecuencia, se mejoró el aprovechamiento de inversiones en equipamiento que, en muchos casos, es de alto costo y con alto índice de obsolescencia. Es decir, este tipo de proyectos aumentó la eficiencia de la inversión en investigación científica.

Además, la formación del consorcio propició un aumento de escala y visibilidad, brindando mayores posibilidades para competir por otros fondos y atraer el interés de los orga- nismos de regulación y registro de productos farmacéuticos. Como resultado de esto, durante el 2010 el consorcio obtuvo por concurso subsidios de aproximadamente 7 millones de dólares para el desarrollo de anticuerpos monoclonales para combatir el cáncer ${ }^{4}$.

\section{Conclusiones generales}

Aunque la pretensión del presente trabajo es meramente descriptivo, en términos conceptuales la asociatividad público-privada para el desarrollo de conglomerados científicos y tecnológicos es explicable a partir de múltiples enfoques de la sociología, la ciencia política y la economía de la innovación.

Ahora bien, desde el punto de vista del diseño de políticas e instrumentos de promoción de la innovación, la asociatividad públicoprivada adquiere un significativo interés. Tanto para los Estados como para las instituciones científicas tiene suma importancia aumentar la probabilidad de que los resultados de investigación desde el ámbito público puedan ser transferidos a la sociedad, como también que las empresas puedan sostener en el tiempo el esfuerzo innovativo.

Puede decirse que el desarrollo de redes científicas, la promoción de la transferencia tecnológica y la construcción de relaciones de confianza parecen ser antecedentes fundamentales en la formación de un consorcio público-privado.

Así, por un lado, parecen haber evidencias respecto a que la historia de la red tiene notable influencia, tanto en la dinámica del

4 Cfr. Ministerio de Ciencias, Tecnología e Innovación Productiva (2011). "Más de \$20.000.000 para la producción local de medicamentos destinados al tratamiento del cáncer". Argentina. Recuperado de http://www.mincyt.gov.ar/multimedia/archivo/archivos/11_02_22_Se_firm_contrato_para_produccin_de_anticuerpos_monoclonales.pdf 
proyecto escalable como en el tiempo en que pueden esperarse resultados científicos y tecnológicos con potencialidad de transferencia a la sociedad.

Por otro lado, el desarrollo de redes es utilizado para apalancar recursos financieros en las actividades de investigación y desarrollo y para introducirse en las agendas de los organismos de regulación y control.

En el caso tratado en este artículo, la utilización del subsidio permitió consolidar la red, aumentar su influencia y tamaño, acelerar el proceso de transferencia tecnológica y darle un marco de sociedad -consorcio- a la red.

Desde el punto de vista integral, la constitución de un consorcio aumentó la visibi-

\section{ReFERENCiAS}

Chesbrough, H. (2003). Open Innovation: The New Imperative for Creating and Profiting from Technology. Boston: Harvard Business School Press.

David, P. y Foray, D. (2002). Una introducción a la economía y a la sociedad del saber. Revista Internacional de las Ciencias Sociales, 171.

Etzkowitz, H. y Leydesdorff, L. (Eds.) (1997). Universities in the Global Economy: A Triple Helix of University-Industry-Government Relations. Londres: Casell Academic.

Freeman, C. (1982). Technological Infrastructure and International Competitiveness. Draft Paper Submitted to the OECD ad hoc Group on Science, Technology and Competitiveness. París: OCDE. lidad internacional, lo que emergió como ventaja al momento de relacionarse con otros grupos y consorcios internacionales. De igual manera, la implementación y/o consolidación del consorcio permitió la formación de profesionales en ámbitos interdisciplinarios y la circulación de personas y conocimientos.

En síntesis, el desarrollo de la red públicaprivada, combinada con el acceso a financiación específica para desarrollar el consorcio, tuvo efectos significativos en el abordaje de problemas más complejos y de mayores requerimientos y en el aumento de la probabilidad de transferencia de los resultados de investigación a la sociedad.

Freeman, C. (1987). Technology Policy and Economic Performance. Lessons from Japan. Londres: Frances Pinter.

Gibbons, M. et ál. (1997). La nueva producción del conocimiento. Madrid: Ediciones Pomares-Corredor

Jansen, D. (2007). Governance - An Integrated Theory. En Jansen, D. (Ed.) New Forms of Governance in Research Organizations. Dordrecht (Holanda): Springer.

Johansson, B., Karlsson, C. y Backman, M. (2007). Innovation Policy Instruments. Electronic Working Paper Series, 105.

Lemarchand, G. (2010). Sistemas nacionales de ciencia, tecnología e innovación en América Latina y el Caribe. Montevideo: UNESCO

López, A. y Lugones, G. (1997). El proceso de innovación tecnológica en América Latina en los 90. REDES, 9. 
Lundvall, B.-Å. (1985). Product Innovation and User-Producer Interaction. Aalborg: Aalborg University Press.

Lundvall, B.-Å. (1988). Innovation as an Interactive Process: From User-Producer Interaction to the National Innovation Systems. En G. Dosi, et ál. (Eds.) Technical Change and Economic Theory. Londres: Pinter Publishers.

Nelson, R. y Winter, S. (1982). An Evolutionary Theory of Economic Change. Harvard University Press.

Nelson, R. (Ed.) (1993). National Innovation Systems: A Comparative Analysis. Nueva York: Oxford University Press.

Nelson, R. (1995, marzo). Recent Evolutionary Theorizing about Economic Change. Journal of Economic Literature, 23, 48-90.

OCDE (2005). Manual de Oslo. Guía para la recogida e interpretación de datos sobre innovación. OCDE-Eurostat.
Pisano, G. (2006). Science Business. The Promise, the Reality and the Future. Harvard Business School Press.

Sábato, J. y Botana, N. (1970). La ciencia y la tecnología en el desarrollo de América Latina. En Herrera, A., et ál. América Latina: ciencia y tecnología en el desarrollo de la sociedad. Santiago de Chile: Editorial Universitaria.

Secretaría de Ciencia, Tecnología e Innovación Productiva (SECyT) (2006). Plan Estratégico Nacional de Ciencia, Tecnología e Innovación "Bicentenario" (2006-2010). Argentina. Recuperado de: http://www. agencia.gov.ar/convocatorias/documentosconvocatorias/plan_estrategico_bicentenario_vp_10jul.pdf

Teubal, M. (1996). R\&D and Technology Policy at NICs as Learning Processes. World Developtmen, 24(3), 449-460.

Teubal, M. (1997). A Catalytic and Evolutionary Approach to Horizontal Technology Policies. Research Policy, 25(8), 1161-1188. 